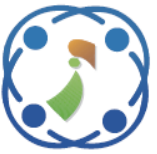

\title{
Diagnosis of Alzheimer Disease Using Fast Independent Component Analysis and Otsu Multi-level Thresholding
}

\author{
Halebeedu Subbaraya Suresha ${ }^{1 *}$ \\ Srirangapatna Sampathkumaran Parthasarathy ${ }^{2}$ \\ ${ }^{1}$ Don Bosco Institute of Technology, India \\ ${ }^{2}$ Professionally Excellent Socially Committed Engineers College of Engineering Mandya, India \\ * Corresponding author's Email: suresha.phd2018@ gmail.com
}

\begin{abstract}
Detection of Alzheimer disease using Magnetic Resonance Imaging (MRI) is the most challenging aspect in the field of medical image processing and analysis. In this paper, the proposed methodology has three major steps: image acquisition, image pre-processing and segmentation. Initially, the brain images were acquired from the dataset: Open Access Series of Imaging Studies (OASIS). After image acquisition, image pre-processing was carried out using median filter, it utilized for cutting down the noise and to improve the quality of acquired brain images. Then, segmentation was carried-out using Fast-Independent Component Analysis (Fast-ICA) along with Otsu multilevel thresholding. It was a flexible high level machine learning technique to localize the object in complex template. In experimental analysis, the proposed approach distinguishes the brain MRI tissues: White Matter (WM), CerebroSpinal Fluid (CSF), and Grey Matter (GM) by means of Tanimoto index, similarity index, precision, and recall. The proposed methodology improved the Alzheimer tissue detection up to 15-30\% compared to the existing methods: Band Expansion Process (BEP), ICA and BEP-ICA in terms of precision and recall.
\end{abstract}

Keywords: Cerebro spinal fluid, Fast independent component analysis, Grey matter, Otsu multi-level thresholding, White matter.

\section{Introduction}

Alzheimer disease is the common neurodegenerative progressive disorder and it is classified by the deterioration of memory and other cognitive functions [1]. In recent decades, numerous neuroimaging techniques employed widely in clinical diagnosis and Alzheimer detection [2]. Generally, two types of detection systems exist in an Alzheimer detection, one is Whole Brain based Detection (WBD) and the other is a Single Slice based Detection (SSD) [3]. The construction of a realistic model requires accurate segmentation of MRI tissue classes based on dissimilar conductivity values [4]. Whereas, the segmentation of brain includes five tissue classes: WM, GM, CSF, skull, and skin [5]. Manually identifying and segmenting the tissue classes within a brain image is naturally insignificant [6]. Manual recognition takes more time for segmenting the tissue classes and also it is applicable for a limited number of brain images [7]. The manual detection of MRI tissue classes is challenging, expensive, time-consuming and inconsistent due to inter-rater and intra-rater variability. So, the automatic detection methodology made an attention within the researcher's community due to its several functional applications [8].

The automated segmentation system can detect tissue classes effectively with high sensitivity and specificity $[9,10]$. In this experimental research, an effective automated system developed, which has of three steps: pre-processing, skull removal and segmentation. Initially, the brain images acquired from the OASIS database. After the acquisition of brain images, median filtering employed for denoising or enhancing the quality of brain images. Whereas, the median filter is a non-linear filter, which is utilized to identify the area of edges in brain images, since non-linear filters are very 
sensitive to noise. The respective pre-processed brain images used for skull removal using Sobel edge detection and dilation morphological operator. The developed methodology delivered an approximation to the gradient magnitude, which helped to detect the edges easily in gray-scale images. After skull removal, segmentation is performed using Fast-ICA along with Otsu multilevel thresholding approach. The fundamental advantage of the proposed segmentation approach is very robust to binary values, which helps to decrease the computational complexity.

This paper is composed as follows. Section 2 survey several recent papers on Alzheimer segmentation strategies. In section 3, an effective segmentation method: Fast-ICA is presented with Otsu multi-level thresholding approach. Section 4 shows comparative experimental result for proposed and existing segmentation strategies using OASIS dataset. The conclusion is made in section 5 .

\section{Literature review}

Several research techniques suggested by researchers in Alzheimer detection. In this scenario, a brief evaluation of some essential contributions to the existing literatures presented in this section.

H. Aidos, and A. Fred, [11] proposed a new methodology to achieve an efficient CAD system for detecting Alzheimer based on Fluoro Deoxy Glucose Positron Emission Tomography (FDGPET) scans. The developed CAD system tried to determine the region of interest and segment all the Alzheimer regions in FDG-PET scans. Then, combined the segmented outcome of CAD system used for identifying the coherent information on all subjects. The developed methodology considered the longitudinal information of the subjects for improving the classification result. While using a large number of features, a perceivable over-fitting of the model was observed that decreases the accuracy of the training set.

Y. K. Jang, C. H. Lyoo, G. D. Rabinovici, S. J. Oh, H. Cho, M. Oh, and W. J. Jagust, [12] performed a head to head comparison of two tracers (AV-1451 and THK5351) in Alzheimer detection and fronto-temporal dementia cases and also investigated, which tracer was suited for tau image in these disorders. This method utilized the standardized uptake value ratio, which divides the mean activity of target volume interest by mean activity of cerebellar hemisphere gray matter. Although, the uptake of two tracers highly correlated, AV-1451 cortical uptake was more striking in Alzheimer detection, where THK5351 cortical uptake showed more prominent in fronttemporal dementia. The AV-1451 was more specific and sensitive to Alzheimer detection type tau and showed lower off-target binding, while THK5351 reflected non-specific Neuro-degeneration. The limitation of the developed methodology was these two traces did not have pathological findings and the number of cases were small, so the findings needed to be replicated in larger samples.

M. Liu, D. Zhang, and D. Shen, [13] developed a new multi-template learning approach for automatic Alzheimer diagnosis using multi-template data. The developed methodology extracted several feature representations from multiple selected templates. After the selection process, a feature selection algorithm was utilized for reducing the dimensionality of the feature vectors in each template. Then, a classification strategy: multiple Support Vector Machine (SVM) was employed to make a final decision. This method achieved significant performance in multi-template based Alzheimer detection /Mild Cognitive Impairment (MCI) classification compared to the other existing methods. This method has high computational cost, because it considers multiple templates and also lack in spatial correlation relationships among the templates.

G. H. Jahng, D. K. Lee, J. M. Lee, H. Y. Rhee, and C. W. Ryu, [14] investigated the Double Inversion Recovery (DIR) images to show the variations of Gray Matter Volume (GVM) between the Alzheimer patients and also to determine the alterations of GVM between the groups using DIR images and T1-weighted (T1W) images. In GMV, the group differences among Cognitively Normal $(\mathrm{CN}), \mathrm{MCI}$ and Alzheimer detection patients were tested by voxel-wise. The DIR images were very sensitive to determine the GVM loss in Alzheimer patients compared to $\mathrm{CN}$ and MCI subjects. The thickness of the slice was too thick for determining the GVM in the brain cortex and increases the voxel resolution scan duration, which produce motion related-artifact in Alzheimer detection patients.

J. Zhang, M. Liu, L. An, Y. Gao, and D. Shen, [15] presented a landmark-based feature extraction method using longitudinal structural MRI images. Compared to the existing approaches, this method avoided the time-taking steps of both non-linear registration and tissue segmentation in the application stage. Initially, the discriminative landmarks were identified from the brain images and then localized the land-marks effectively by employing fast land-mark detection algorithm. The high-level contextual longitudinal and statistical 


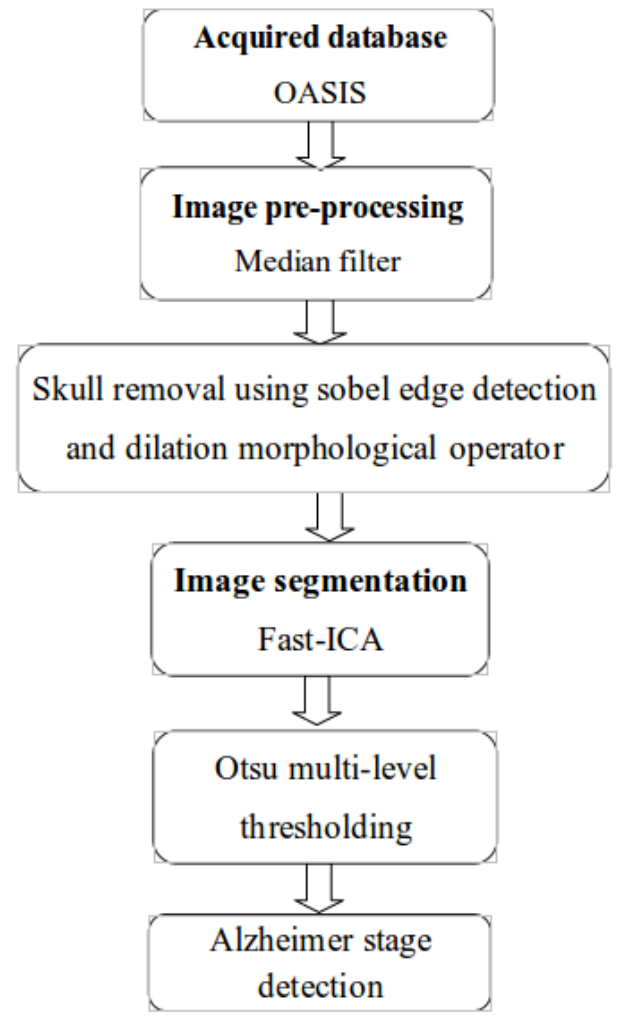

Figure. 1 Working procedure of proposed methodology

spatial features were extracted from the detected land-marks. A linear SVM approach was adopted to distinguish Alzheimer detection/MCI subjects from healthy controls by using these features. The developed method achieved superior classification performance in both Alzheimer detection and MCI classification. The learning performance of the subsequent classification model was affected by the noise or redundant features.

An unsupervised algorithm (Fast ICA with Otsu multi-level thresholding segmentation approach) implemented to overcome the above mentioned drawbacks and additionally enhance the performance of Alzheimer tissue segmentation and detection.

\section{Proposed methodology}

The proposed Alzheimer detection system has four steps: image acquisition, image pre-processing, skull removal and segmentation. The proposed Alzheimer detection system block diagram represented in the Fig. 1. The brief description about the proposed technique described above.

\subsection{Image acquisition}

In the initial stage of the Alzheimer detection system, the brain images are taken from the standard
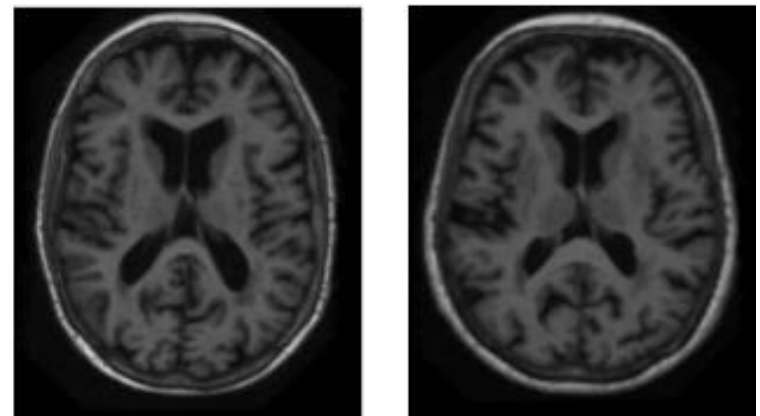

Figure. 2 Sample acquired brain image

benchmark dataset: OASIS database. The original OASIS dataset contains 1500 raw image scans, which is captured from 609 normal adults and 489 persons at different stages of cognitive decline ranging in age from 42-95 years. This database consists of 2000 MR sessions that comprises of susceptibility weighted imaging, arterial spin labelling, diffusion tensor imaging sequences, time of flight, etc. After obtaining the brain images, an important step (pre-processing) is carried out using median filter. The sample acquired brain image is denoted in the Fig. 2.

\subsection{Image pre-processing}

After the acquisition of brain images, median filter is utilized as the pre-processing methodology. Median filter is a non-linear filter, which belongs to the edge preserving smoothing filter classes. The median filter easily smooth's the data and also it contains the sharp details about the smoothed data. The median is the middle value of all neighbourhood pixel values. The median value is a stronger "central indicator" than the average value. The mathematical equation to calculate the middle value represented in the Eq. (1).

$$
\underset{\operatorname{median}[B(x)]}{\operatorname{median}[A(x)+B(x)] \neq \operatorname{median}[A(x)]+}
$$

Where, $A(x)$ and $B(x)$ are the two different brain images.

In specific, the median is highly affected by a discrepant small number among the neighbourhood pixels. Therefore, median filter is very significant in eliminating several types of noises. The median filter considers each pixel in the brain image for deciding whether it is a representative of its surroundings or not. Instead of changing the pixel value with neighbourhood mean pixel values, the median filter changes the pixel value with middle pixel values. The median is determined by separating the pixel values of neighbouring pixels in 



Figure. 3 Pre-processed brain image

a numerical order. Then, inter-change the pixel value with middle pixel value. If the neighbourhood pixel contains an even number of pixels, the average of two middle pixel values is utilized. The sample of pre-processed brain image is represented in the Fig. 3.

\subsection{Skull removal}

In this section, the pre-processed brain image used for skull removal by employing Sobel edge detection and dilation morphological operator. The Sobel edge detection methodology delivers an approximation to the gradient magnitude, which helps to easily detect the edges in gray-scale images. In addition, the dilation operator probes and expands the shapes contained in the input image that is very useful in skull removal. The brief description about the undertaken methodologies described below.

\subsubsection{Sobel edge detection}

The Sobel edge detection approach works on the basis of edges. Using the edges, determine the derivative intensity value of the brain images and identify the points, where the derivatives are high, then the edges are located. In gradient vector, the components help to measure the rapid pixel value, that change on the basis of $x$ and $y$ direction. Thus, the gradient components are determined by employing the Eqs. (2) and (3).

$$
\begin{aligned}
& \Delta x=\frac{f(x+d x, y)-f(x, y)}{d x} \\
& \Delta y=\frac{f(x, y+d y)-f(x, y)}{d y}
\end{aligned}
$$

Where, $d x$ and $d y$ represented as the distance measure of $x$ and $y$ directions. In discrete brain images, the numbers of pixels between the two points $d x$ and $d y$. In this scenario, $d x=d y=1$ is the pixel spacing point in which the pixel coordinates $(i, j)$ are calculated by employing the Eqs. (4) and (5).

$$
\begin{aligned}
\Delta x & =f(i+1, j)-f(i, j) \\
\Delta y & =f(i, j+1)-f(i, j)
\end{aligned}
$$

To identify the presence of gradient discontinuity, determine the changes in gradient at $(i, j)$, which is done by identifying the magnitude measure by employing the Eq. (6).

$$
M=\sqrt{\Delta x^{2}+\Delta^{2} y}
$$

The gradient direction $\theta$ is calculated by using the Eq. (7)

$$
\theta=\tan ^{-1}\left[\frac{\Delta y}{\Delta x}\right]
$$

The Sobel edge detection methodology gives more attention on the brain image pixels, especially which are closer to the centre of the mask. This method detects the edges, where the gradient magnitude is higher in gray-scale images, which are named as absolute gradient magnitude.

\subsubsection{Pseudo-code for sobel edge detection method}

Input: Consider sample brain image

Output: Detect the edges

Step 1: Acquire the input brain image

Step 2: Employ mask in the input image

Step 3: Sobel edge detection approach is employed to detect the gradient

Step 4: Mask manipulation is done separately for the objects in the input brain image

Step 5: Experimental outcomes are combined to determine the absolute gradient magnitude

Step 6: Absolute magnitude is represented as the output edges.

\subsubsection{Morphological operation using dilation}

Dilation is one of the basic operators in mathematical morphology, it is mainly used in the gray-scale images. The dilation operator gradually increases the effect of foreground pixel boundaries, so the neighbourhood areas and holes in the region become smaller. Dilation operator has two phases, first phase is the dilated input image and the second phase is the structuring element called as the kernel. This structuring element is used to determine how much the acquired image is dilated. The mathematical equation of dilation is represented in the Eq. (8).

$$
A \oplus B=\left\{X /\left(\hat{B_{X}}\right) \cap A \neq \Phi\right\}
$$





Figure. 4 Skull removed brain image

Consider, $A$ is the input image co-ordinates, $B$ is the structuring element co-ordinates and $\Phi$ is represented as the null operator. The graphical representation of the skull removed brain image is represented in the Fig. 4.

\subsection{Segmentation}

Image segmentation is defined as the procedure of categorizing the brain image into dissimilar regions that are homogeneous with respect to some image features: colour, texture, shape, etc. The goal of image segmentation is to extract and recognize a particular region in an acquired image. In this experimental research, fast-ICA along with Otsu multi-level thresholding methodology is undertaken for image segmentation. The main aim of the proposed segmentation approach is to extract the suspicious area in brain images. Suspicious region in the brain image has more false positives and high sensitivity, which are effectively determined by the proposed segmentation algorithm.

\subsubsection{Fast independent component analysis}

Generally, ICA is a linear transformation method that helps to separate the multi-variate components into additive sub-components. Whereas, the additive sub-components are non-Gaussian approach that are statistically free from the other independent scheme. The ICA helps to solve the higher order data and also determines the independent source point for the linear mixture components. Hence, ICA delivers a significant data representation by applying image decomposition and representation. Usually, an effective algorithm is employed to estimate the independent components, which is very tough to define the energies and the order of independent components. To overcome this issue, several ICA algorithms proposed that differ in their objective functions for statistical independence.

For the fast computation and efficient data reduction, Fast-ICA method is used. Fast-ICA is an adequate and well-known algorithm for ICA, which is based on a fixed-point reiteration approach. This algorithm maximizes the non-Gaussian statistical independent measure. Here, vast collections of data points are utilized in a single step of the algorithm that makes the computation faster. Fast-ICA algorithm includes pre-processing operations like centering, and whitening the data. The brain image is centered by computing the mean of each component in the image. Whitening is the procedure of linearly transforming the data into new components. The output image of fast-ICA is given as the input for Otsu multi-level thresholding methodology.

\subsubsection{Otsu multi-level thresholding}

The Otsu multi-level thresholding is based on the discriminate analysis for identifying the maximum separability of classes and also it is utilized to perform automatic histogram shape based image thresholding. Initially, the Otsu multi-level thresholding algorithm assumes the pre-processed brain image, which contains two classes: background $C_{1}$ and foreground $C_{0}$. The Otsu multilevel thresholding constructs a normalized histogram using discrete probability density function, which is represented in the Eq. (9).

$$
p_{r}\left(r_{q}\right)=\frac{n_{q}}{n}, q=0,1,2, \ldots \ldots, L-1
$$

Where, $n$ is denoted as total number of pixels in the brain image, $n_{q}$ is represented as the pixel that has an intensity level $r_{q}, L$ is the highest intensity level of the image.

Initial threshold is the mid-point between the minimum and maximum intensity value of the brain image. If $k$ is chosen as the initial threshold, then $C_{0}$ is the set of pixels with levels $[0,1, \ldots k-1]$ and $C_{1}$ is the set of pixels with levels $[k, k+1, \ldots L-$ 1].Otsu multi-level algorithm chooses the threshold value $k^{*}$ which maximizes the between class variance $\sigma^{2} B$ that is defined in the Eq. (10).

$$
\sigma^{2} B=w_{0}
$$

Where,

$$
w_{0}=\sum_{q=0}^{k-1} p_{q}\left(r_{q}\right)
$$

Therefore, the optimal threshold value $k^{*}$ is obtained by using the Eq. (12),

$$
\sigma^{2} B\left(k^{*}\right)=\frac{\arg }{0<k<L-1} \max \sigma^{2} B(k)
$$


Table 1. Segmented outcome of proposed methodology

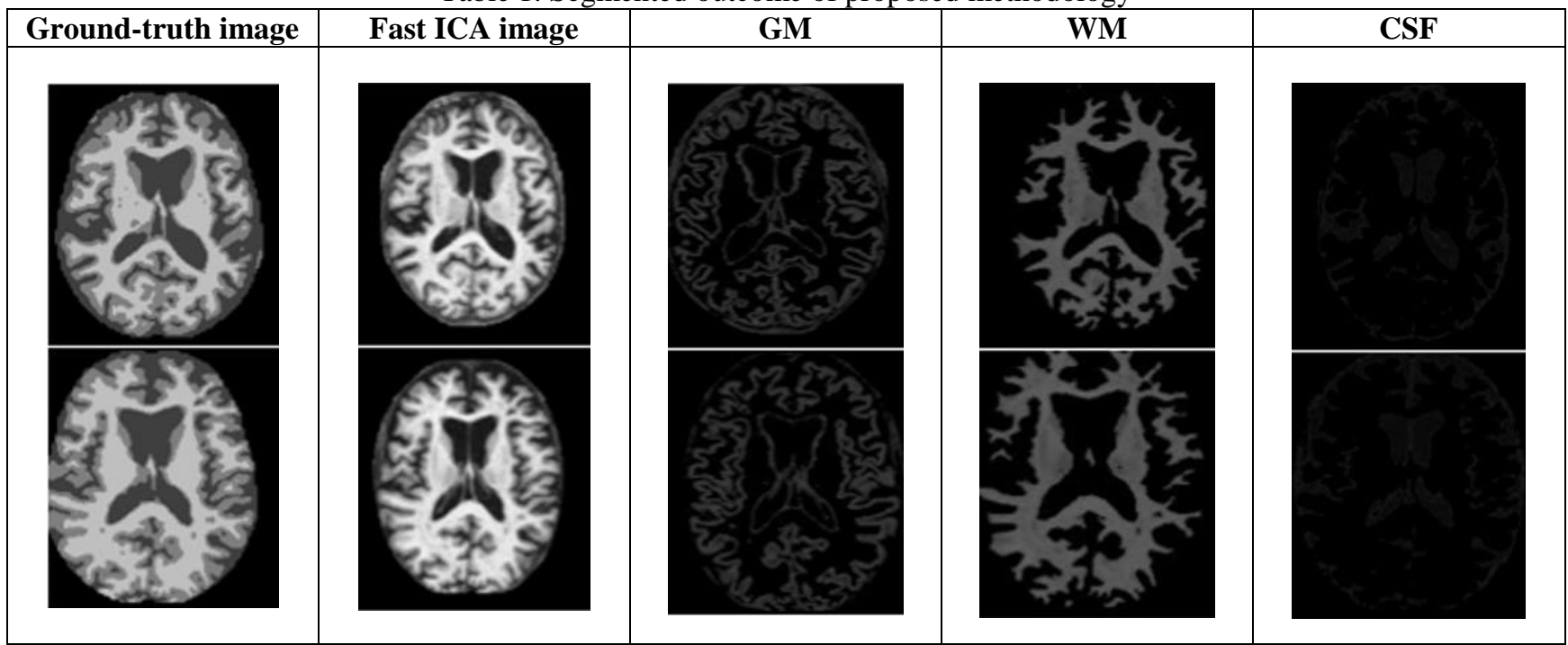

Though, Otsu multi-level thresholding is the most widely utilized thresholding algorithm for several applications by maximizing the class variance in gray-scale brain image. The obtained threshold value achieves satisfactory result for several medical imaging applications. The graphical representation of the segmented brain image is represented in the Table 1. Finally, the segmented output is compared with the ground truth image for validating the efficiency of the proposed methodology.

\section{Experimental analysis}

For experimental simulation, MATLAB (version 2017a) was employed on PC with $3.2 \mathrm{GHz}$ with i5 processor. In order to estimate the efficiency of the proposed algorithm, the performance of the proposed method was compared with BEP, BEPICA, and ICA on the reputed database: OASIS. The performance of the proposed methodology was compared in terms of Tanimoto index, similarity index, precision, and recall.

\subsection{Performance measure}

Performance measure is defined as the relationship between the input and output variables of a system understood by employing the suitable performance metrics like precision and recall. The general formula for calculating the precision and recall of Alzheimer detection are mathematically represented in the Eqs. (13) and (14).

$$
\begin{aligned}
& \text { Precision }=\frac{T P}{(T P+F P)} \times 100 \\
& \text { Recall }=\frac{T P}{(T P+F N)} \times 100
\end{aligned}
$$

Additionally, for segmentation validation, the similarity index is expressed in terms of TP, TN, FP and FN counts, which is obtained by matching the segmented result to the ground truth image. The general formula utilized to calculate the similarity index [16] is represented in the Eq. (15).

$$
\text { Similarity index }=\frac{2 T P}{(2 T P+F P+F N)}
$$

Where, the similarity index value " 0 " shows no similarity between the results and the value " 1 " demonstrates the similarity between the output and ground truth image. In Tanimoto index, the TP values detected by the overlaps between the manually segmented ground truth tumour labels and the machine generated tumour labels. The general formula used to calculate Tanimoto index [16] is denoted in the Eq. (16).

$$
\text { Tanimoto index }=\frac{T P}{F P+F N+T P}
$$

Where, $T P$ is represented as true positive, $F P$ is denoted as false positive, $T N$ is stated as true negative and $F N$ is specified as false negative.

\subsection{Experimental analysis on OASIS dataset}

In this experimental analysis, OASIS dataset is used for comparing the performance evaluation of existing methods: BEP, ICA and BEP+ICA and the proposed scheme: Fast ICA-Otsu multi-level thresholding segmentation approach. In Table 2, the performance evaluation of existing and proposed method is validated in terms of Tanimoto index and similarity index. The acquired brain image consists of three tissue classes: WM, GM and CSF. Here, the 
performance evaluation is validated for twelve sample brain images in each tissue classes. The validation result showed that the proposed method outperformed the existing methodologies in terms of Tanimoto index and similarity index.

For 12 medical brain images, the average Tanimoto index of the proposed technique Fast ICA-Otsu multi-level thresholding approach delivered 0.64 and the existing methodologies BEP, ICA and BEP+ICA delivered 0.3 and 0.4 and 0.5 of Tanimoto index respectively. Similarly, the average similarity index of the proposed technique delivers 0.77 and the existing methodologies delivers 0.41 , 0.51 and 0.57 of similarity index respectively. The graphical representation of average Tanimoto index and similarity index presented in the Figs. 5 and 6.

Table 2. Tanimoto and similarity index comparison of proposed and existing methods

\begin{tabular}{|c|c|c|c|c|c|c|c|c|}
\hline \multirow{2}{*}{ Methodologies } & \multicolumn{4}{|c|}{ Tanimoto index } & \multicolumn{4}{c|}{ Similarity index } \\
\cline { 2 - 9 } & CSF & GM & WM & Average & CSF & GM & WM & Average \\
\hline BEP [16] & 0.10 & 0.49 & 0.25 & 0.3 & 0.18 & 0.66 & 0.40 & 0.41 \\
\hline ICA [16] & 0.3 & 0.6 & 0.24 & 0.4 & 0.43 & 0.73 & 0.39 & 0.51 \\
\hline BEP+ICA [16] & 0.4 & 0.71 & 0.3 & 0.5 & 0.51 & 0.83 & 0.38 & 0.57 \\
\hline Proposed & 0.59 & 0.54 & 0.80 & 0.64 & 0.74 & 0.70 & 0.89 & 0.77 \\
\hline
\end{tabular}

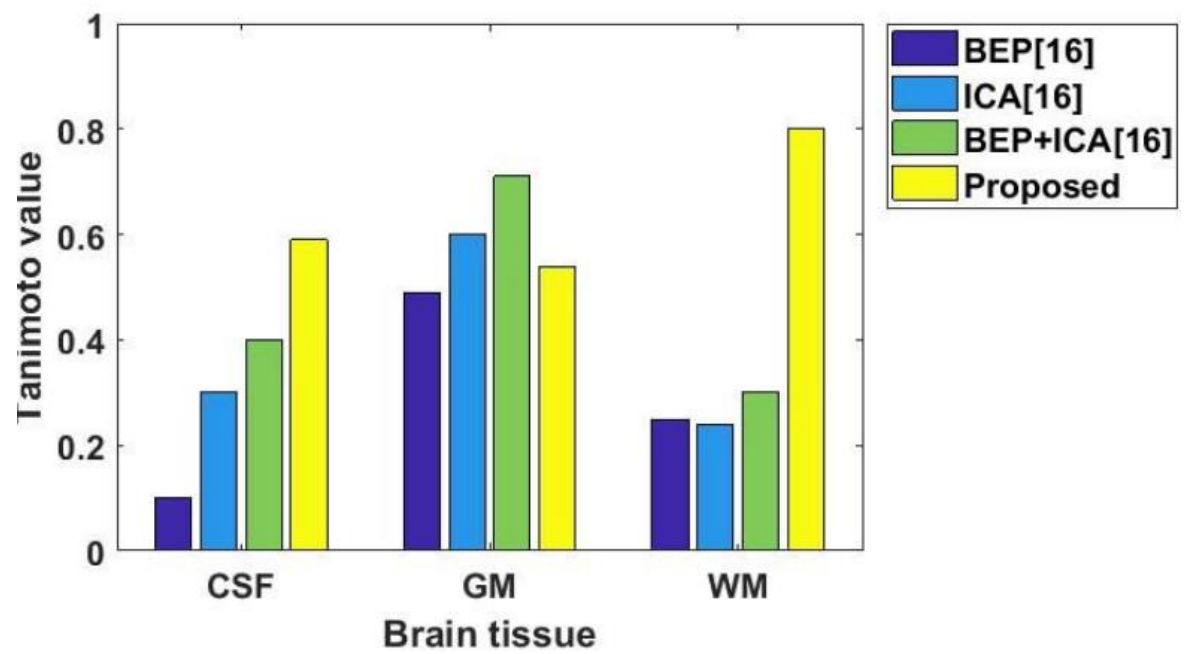

Figure. 5 Graphical representation of average tanimoto index

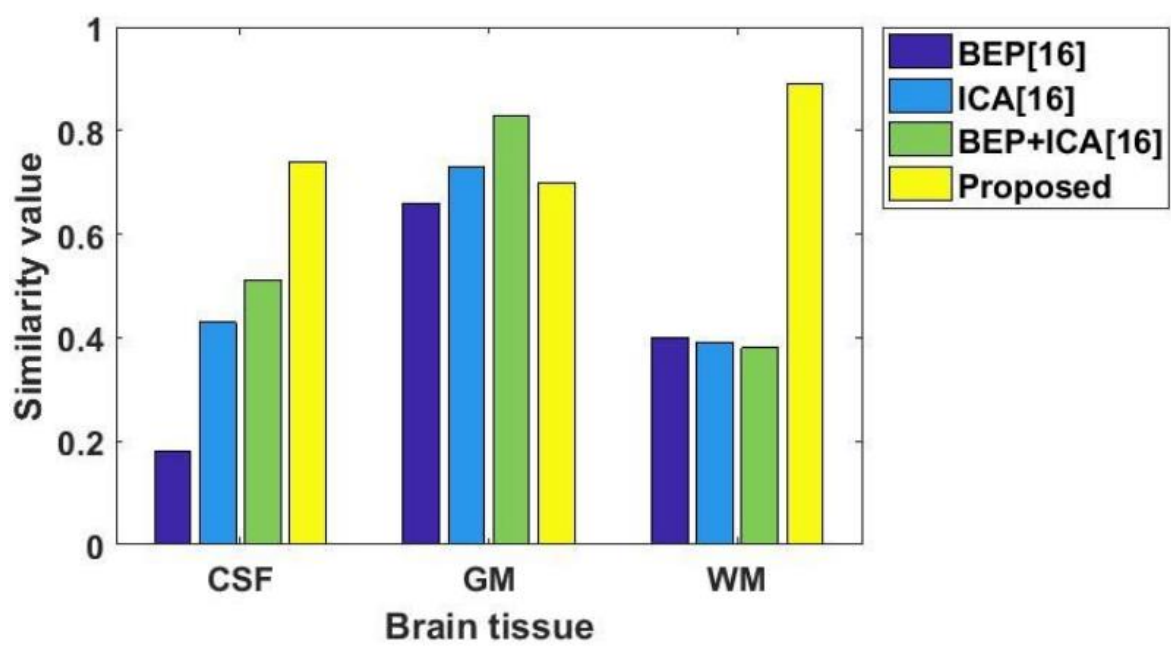

Figure. 6 Graphical representation of average similarity index 
Table 3. Precision and recall comparison of proposed and existing methods

\begin{tabular}{|c|c|c|c|c|c|c|c|c|}
\hline \multirow{2}{*}{ Methodologies } & \multicolumn{4}{|c}{ Precision (\%) } & \multicolumn{4}{c|}{ Recall (\%) } \\
\cline { 2 - 10 } & CSF & GM & WM & Average & CSF & GM & WM & Average \\
\hline BEP [16] & 13 & 55 & 27 & 32 & 30 & 85 & 27 & 47 \\
\hline ICA [16] & 41 & 68 & 96 & 68 & 41 & 81 & 20 & 47 \\
\hline BEP+ICA [16] & 41 & 94 & 89 & 74 & 69 & 94 & 25 & 62 \\
\hline Proposed & 77 & 100 & 86 & 87 & 64 & 80 & 94 & 79 \\
\hline
\end{tabular}

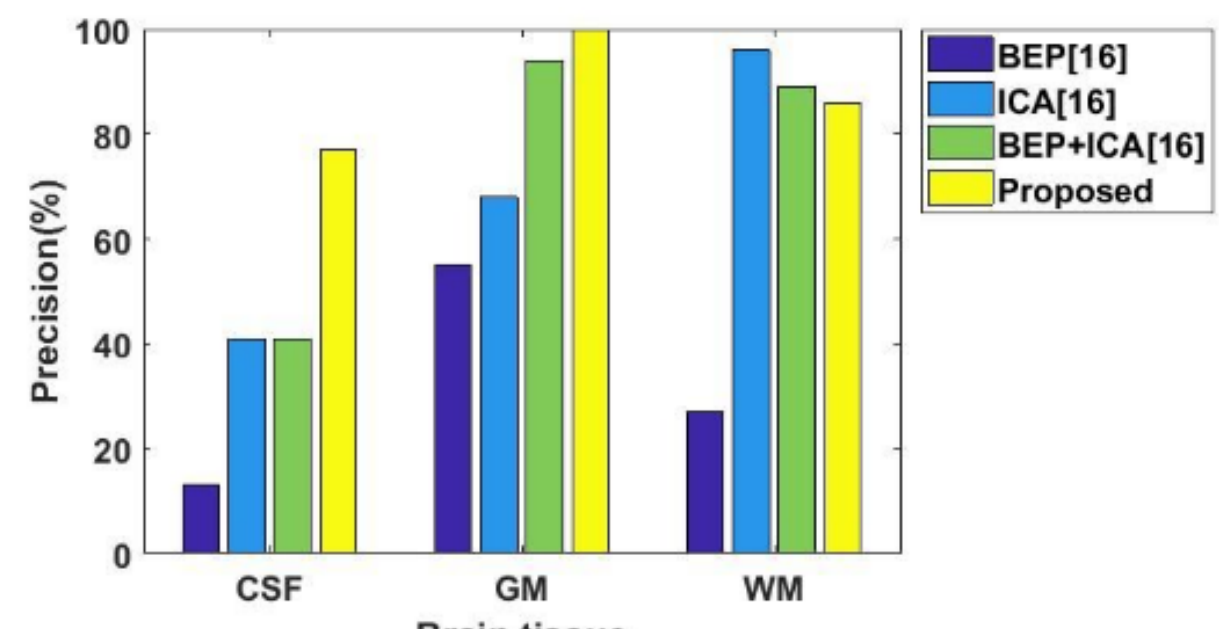

Brain tissue

Figure. 7 Graphical representation of average precision value

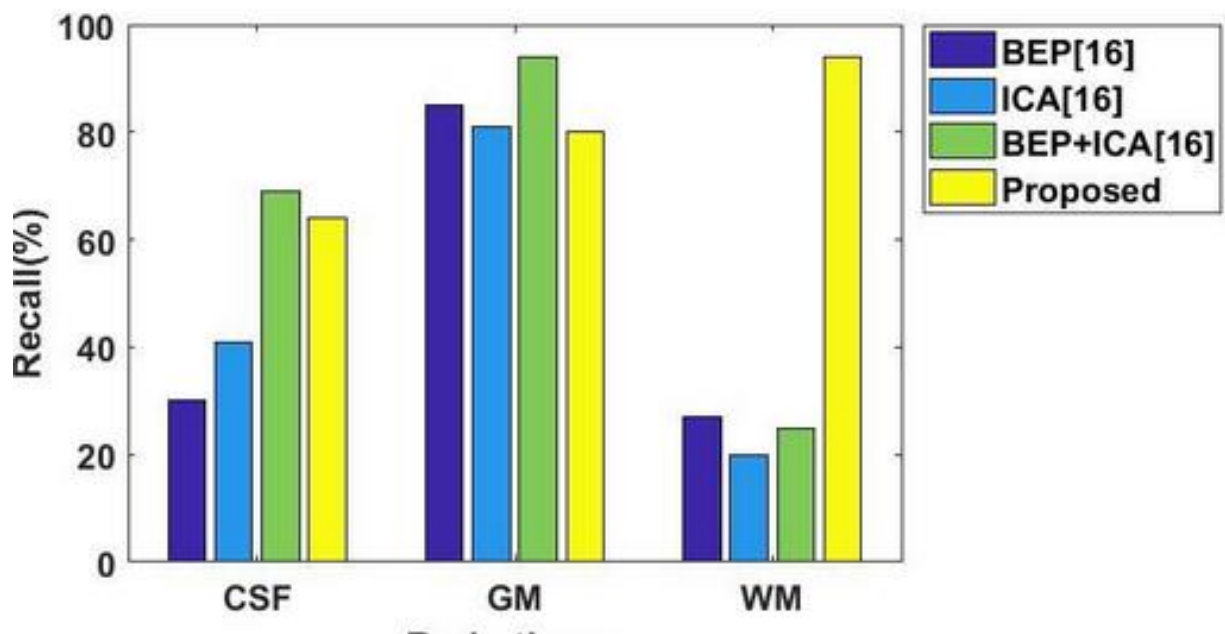

Brain tissue

Figure. 8 Graphical representation of average recall value

In Table 3, the performance evaluation of proposed and existing methods validated in terms of recall and precision. The average precision value of the proposed technique Fast ICA-Otsu multi-level thresholding approach delivered $87 \%$ and the existing methodologies BEP, ICA and BEP+ICA delivered $32 \%$ and $68 \%$ and $74 \%$ of precision value, respectively. The graphical representation of average precision value presented in the Fig. 7.

In addition, the average recall value of the proposed technique delivered $79 \%$ and the existing methodologies delivered $47 \%, 47 \%$ and $62 \%$ of recall value, respectively. The graphical representation of average recall value presented in the Fig. 8. The Tables 2 and 3 confirmed that the proposed methodology performed better than the existing methods on the OASIS dataset, because Fast ICA-Otsu multi-level thresholding approach encodes the both texture and shape features of brain images for improving the segmentation efficiency of Alzheimer. The Fast ICA-Otsu multi-level thresholding algorithm fuse different pixel information, which represents different correlation space to produce a new correlation value. 


\section{Conclusion}

Alzheimer detection is one of the most significant research tasks in computer-aided health monitoring system. The objective of the research work is to develop a proper segmentation methodology for segmenting the brain MRI tissues like CSF, WM and GM using OASIS dataset. In this scenario, fast-ICA segmentation approach along with Otsu multi-level thresholding used to segment the MRI brain tissues. The major advantages of fastICA segmentation methodology are, less computational cost, and robustness to preserve brain image details. Otsu multi-level thresholding employed on the segmented output to find the best threshold value accurately and quickly for extracting the exact object from background image. Compared to other obtainable approaches in Alzheimer detection, the proposed scheme delivered an effective performance in terms of Tanimoto index, similarity index, precision, and recall. The proposed methodology showed $15-30 \%$ of enhancement in segmentation than the existing methods by means of precision and recall. In the future work, for classifying the stages of detected brain tissues, descriptor level feature extraction methodologies will be employed with an appropriate multiobjective classification method and also an effective approach will be developed for accurate skull removing from brain images.

\section{References}

[1] Q.Y. Zhu, S.W. Bi, X.T. Yao, Z.Y. Ni, Y. Li, B. Y. Chen, and X.L. Shang, "Disruption of thalamic connectivity in Alzheimer's disease: a diffusion tensor imaging study", Metabolic Brain Disease, Vol.30, No.5, pp.1295-1308, 2015.

[2] S.H. Wang, Y. Zhang, Y.J. Li, W.J. Jia, F.Y. Liu, M.M. Yang, and Y.D. Zhang, "Single slice based detection for Alzheimer's disease via wavelet entropy and multilayer perceptron trained by biogeography-based optimization", Multimedia Tools and Applications, pp.1-25, 2016.

[3] F.J. Martínez-Murcia, J.M. Górriz, J. Ramírez, C.G. Puntonet, and I.A. Illán, "Functional activity maps based on significance measures and independent component analysis", Computer Methods and Programs in Biomedicine, Vol.111, No.1, pp.255-268, 2013.

[4] Q. Mahmood, A. Chodorowski, A. Mehnert, J. Gellermann, and M. Persson, "Unsupervised Segmentation of Head Tissues from Multi- modal MR Images for EEG Source Localization", Journal of Digital Imaging, Vol.28, No.4, pp.499-514, 2014.

[5] M. Dadar, T.A. Pascoal, S. Manitsirikul, K. Misquitta, C. Tartaglia, J. Brietner, and D.L. Collins, "Validation of a Regression Technique for Segmentation of White Matter Hyperintensities in Alzheimer's Disease", IEEE Transactions on Medical Imaging, Vol.36, No.8, pp.1758-1768 2017.

[6] T.M. Schouten, M. Koini, F. de Vos, S. Seiler, M. de Rooij, A. Lechner, and S.A. Rombouts, "Individual classification of Alzheimer's disease with diffusion magnetic resonance imaging", Neuro Image, Vol.152, pp.476-481, 2017.

[7] R.T. Dietmar, "Symptomatic and preclinical Alzheimer's disease: Neuropathology and imaging", Neurology, Psychiatry and Brain Research, Vol.22, No.2, pp.127-131, 2016.

[8] K.E. McAleese, L. Walker, S. Graham, E.L. Moya, M. Johnson, D. Erskine, and A. J. Thomas, "Parietal white matter lesions in Alzheimer's disease are associated with cortical neurodegenerative pathology, but not with small vessel disease", Acta neuropathologica, Vol.134, No.3, pp.459-473, 2017.

[9] K. Chiotis, L. Saint-Aubert, I. Savitcheva, V. Jelic, P. Andersen, J. Eriksson, and A. Nordberg, "Imaging in-vivo tau pathology in Alzheimer's disease with THK5317 PET in a multimodal paradigm", European Journal of Nuclear Medicine and Molecular Imaging, Vol.43, No.9, pp.1686-1699, 2016.

[10] T. Tuszynski, M. Rullmann, J. Luthardt, D. Butzke, S. Tiepolt, H. J. Gertz, and H. Barthel, "Evaluation of software tools for automated identification of neuroanatomical structures in quantitative $\beta$-amyloid PET imaging to diagnose Alzheimer's disease", European Journal of Nuclear Medicine and Molecular Imaging, Vol.43, No.6, pp.1077-1087, 2016.

[11] H. Aidos and A. Fred, "Discrimination of Alzheimer's disease using longitudinal information", Data Mining and Knowledge Discovery, Vol.31, No.4, pp.1006-1030, 2017.

[12] Y.K. Jang, C.H. Lyoo, G.D. Rabinovici, S.J. Oh, H. Cho, M. Oh, and W.J. Jagust, "HeadTo-Head comparison of [18F] AV-1451 and [18F] THK5351 for tau imaging in Alzheimer's disease and frontotemporal dementia", Alzheimer's \& Dementia: The Journal of the Alzheimer's Association, Vol.13, No.7, pp.1347-1348, 2017.

[13] M. Liu, D. Zhang, and D. Shen, "Relationship induced multi-template learning for diagnosis 
of Alzheimer's disease and mild cognitive impairment", IEEE Transactions on Medical Imaging, Vol.35, No.6, pp.1463-1474, 2016.

[14] G.H. Jahng, D.K. Lee, J.M. Lee, H.Y. Rhee, and C.W. Ryu, "Double inversion recovery imaging improves the evaluation of gray matter volume losses in patients with Alzheimer's disease and mild cognitive impairment", Brain Imaging and Behavior, Vol.10, No.4, pp.10151028, 2016.

[15] J. Zhang, M. Liu, L. An, Y. Gao, and D. Shen, "Alzheimer's Disease Diagnosis using Landmark-based Features from Longitudinal Structural MR Images", IEEE Journal of Biomedical and Health Informatics, Vol.21, No.6, pp.1607-1616, 2017.

[16] R.S. Kamathe, and K.R. Joshi, "A novel method based on independent component analysis for brain MR image tissue classification into CSF, WM and GM for atrophy detection in Alzheimer's disease", Biomedical Signal Processing and Control, Vol.40, pp.41-48, 2018. 